\title{
Noncommutative optimal control and quantum networks
}

\author{
Masahiro Yanagisawa \\ Control and Dynamical Systems, California Institute of Technology, Pasadena, California 91125, USA
}

(Received 17 October 2005; published 24 February 2006)

\begin{abstract}
Optimal control is formulated based on a noncommutative calculus of operator derivatives. The use of optimal control methods in the design of quantum systems relies on the differentiation of an operator-valued function with respect to the relevant operator. Noncommutativity between the operator and its derivative leads to a generalization of the conventional method of control for classical systems. This formulation is applied to quantum networks of both spin and bosonic particles for the purpose of quantum state control via quantum random walks.
\end{abstract}

DOI: 10.1103/PhysRevA.73.022342

PACS number(s): 03.67.-a, 02.30.Yy, 42.50.Dv

\section{INTRODUCTION}

Since the information theoretical structure of quantum systems was analyzed from the engineers' perspective in the 1970s [1,2], many fundamental engineering methodologies have been applied to quantum systems [3]. Applications of control theory have also been considered in many different ways theoretically [4-13] and experimentally [14-20]. A main difference between classical and quantum systems is represented by the noncommutativity of relevant observables and states, which defines distinctive properties of significant information theoretical quantities such as relative entropy. In control theory, however, there are few analyses in which the noncommutativity makes an essential difference $[21,22]$.

In this paper, we formulate optimal control theory by taking the noncommutativity into account based on operator derivatives. A standard method of optimal control for classical systems can be considered along the same line as analytical mechanics [23]. Operator derivatives serve as a basis for the extension of optimal control theory to the quantum domain since the noncommutativity leads to a different law of differentiation. Using this formulation, we investigate the control of quantum networks and continuous time random walks.

Quantum analogues of discrete and continuous time random walks have been considered for quantum computation, communication, and information processing as many classical algorithms are based on random walks. In the discrete case, a random walk is defined by repeated applications of local and global unitary operations. The development of a quantum algorithm is thus related to the design of the discrete unitary operations. It is naturally extended to the continuous case $[24,25]$, in which a random walk is defined by an infinitesimal operator and quantum algorithms are based on the design of a Hamiltonian accordingly. Continuous time quantum walks are used for various engineering purposes: algorithmic speedup for a specific oracular problem [26], quantum state transmission, and entanglement generation over quantum networks [27-32], and so on. These quantum engineering problems are associated with the design of a Hamiltonian to increase the state transition probability along the continuous time quantum random walks.

To utilize quantum networks as communication channels, highly controlled transmission of the quantum state is re- quired $[33,34]$. It is not difficult to design the system to improve the performance if one is allowed to change the global interactions of the networks because it is substantially equivalent to controlling a single system. Difficulties of controlling networks arise from the fact that control manipulations are confined to a few local variables with which we wish to improve the global performance of large degrees of freedom. To this end, we will develop noncommutative optimal control.

Although we will consider the continuous case, it is helpful to illustrate the process of quantum network control using a discrete model of a particle with spin-1/2 on a line. The system is described by a composite Hilbert space representing both spin and position degrees of freedom. Suppose two operators are on the composite space: One is a conditional translation of the particle that shifts the position in the positive direction if the spin is up and vice versa. This is a global operation. The other is a rotation in the spin space, which corresponds to a coin flip. The quantum random walk is defined by alternate applications of these two operators. Note that if the spin degree of freedom is measured at every step, we obtain a classical random walk whose limiting distribution approaches a Gaussian distribution. By contrast, because of the quantum correlations between different positions, the quantum random walk exhibits a broader distribution, which enables faster information transmission than the corresponding classical random walk. In this process, there are degrees of freedom left for us to control the particle. Suppose that we are given a set of spin rotation operators and allowed to pick up one of them to apply at each step. Then, it is possible to increase the potential ability for information transmission by choosing appropriate rotations. It will be shown that noncommutative optimal control provides an effective method to design the spin rotations to improve the global performance.

This paper is organized as follows: Section II provides a mathematical basis of operator derivatives with which noncommutative optimal control theory is formulated and a general solution is given. Section III introduces models of quantum random walks for both spin-1/2 and bosonic systems and optimal control problems are formulated. In Sec. IV, fundamental properties of the quantum random walk are examined. In Sec. V, the optimal control for the quantum random walks is considered and numerical analyses are given. In Sec. VI, we consider further applications of noncommu- 
tative optimal control: an optimal control problem for the bosonic system under measurement and the formulation of quantum noise reduction based on the developed methods.

\section{OPERATOR DERIVATIVES}

We define the differentials of an operator-valued function and a functional with respect to an operator, and formulate noncommutative optimal control.

\section{A. Definitions}

Denote by $\mathcal{L}(\mathcal{H})$, a set of linear operators on a Hilbert space $\mathcal{H}$ and by $\operatorname{Hy}(\mathcal{L})$ a set of hyperoperators. For an operator-valued function $f$ of $X \in \mathcal{L}(\mathcal{H})$, the Gâteaux differential is defined as $[35,36]$

$$
d f(X)=\lim _{h \rightarrow 0} \frac{f(X+h d X)-f(X)}{h},
$$

where $h \in \mathbf{C}$ and $d X \in \mathcal{L}(\mathcal{H})$. The differentiation of $f(X)$ with respect to $X$ is defined by a hyperoperator

$$
\frac{d f(X)}{d X}:=d f(X)(d X)^{-1} \in \operatorname{Hy}(\mathcal{L}) .
$$

For example, if $f(X)=X^{2}, d f(X)=\left(2 X-C_{X}\right) d X$, where $C_{X} \in \operatorname{Hy}(\mathcal{L})$ is defined as $C_{X} \cdot=[X, \cdot]$. The derivative is then given by $d f(X) / d X=2 X-C_{X} \in \mathrm{Hy}(\mathcal{L})$. Note that the first term should be interpreted as the left multiplication hyperoperator $L_{X} \in \operatorname{Hy}(\mathcal{L})$ such that $L_{X} \cdot=X$. In general, for any positive integer $n$, it can be shown by induction that $\left(X-C_{X}\right)^{n} d X=d X X^{n}$, and hence, $d X^{n} / d X=\sum_{j=1}^{n} X^{j-1}\left(X-C_{X}\right)^{n-j}$. This can be rewritten as

$$
\begin{aligned}
\frac{d X^{n}}{d X} & =\sum_{j=1}^{n} \sum_{k=0}^{n-j}\left(\begin{array}{c}
n-j \\
k
\end{array}\right) X^{n-k-1}\left(-C_{X}\right)^{k} \\
& =\sum_{k=0}^{n-1}\left(\begin{array}{c}
n \\
k+1
\end{array}\right) X^{n-k-1}\left(-C_{X}\right)^{k} .
\end{aligned}
$$

As a result, for a polynomial function $f$, we have

$$
\frac{d f(X)}{d X}=\sum_{l=1} \frac{f^{(l)}(X)}{l !}\left(-C_{X}\right)^{l-1}
$$

For example, $d e^{X} / d X=e^{X}\left(1-e^{-C_{X}}\right) C_{X}^{-1}$, where $C_{X}^{-1}$ is a formal notation to simplify the infinite series. In general, denote by $\hat{f}$ the Fourier transform of a function $f$ and define an operator-valued function $f(X)$ as

$$
f(X)=\int d z \hat{f}(z) e^{i z X}
$$

The relation derived above yields [see also Eq. (7)]

$$
\frac{d f(X)}{d X}=\int d z \hat{f}(z) \frac{e^{i z X}-e^{i z\left(X-C_{X}\right)}}{C_{X}}=\frac{f(X)-f\left(X-C_{X}\right)}{C_{X}},
$$

which is equivalent to Eq. (4) for polynomial functions.

By definition, one can easily show the chain rule

$$
\frac{d f(g(X))}{d X}=\frac{d f(g(X))}{d g(X)} \frac{d g(X)}{d X} .
$$

Likewise, if an operator $X(t)$ is parametrized by $t \in \mathbf{R}$,

$$
\frac{d f(X(t))}{d t}=\frac{d f(X(t))}{d X(t)} \frac{d X(t)}{d t} .
$$

Let $J(t)=J(X(t)) \in \mathbf{C}$ be a functional on $\mathcal{L}(\mathcal{H})$ and assume that there exists $d X(t) / d t$. It follows from the Riesz representation theorem that there exists a unique adjoint operator $A$ $\in \mathcal{L}^{*}(\mathcal{H})$ such that

$$
\frac{d J}{d t}=\operatorname{Tr} A^{\dagger} \frac{d X}{d t} .
$$

Then, the differential of $J$ with respect to $X \in \mathcal{L}(\mathcal{H})$ is defined as $d J / d X=A$. For example, when $J(t)=\operatorname{Tr} B^{\dagger} X^{2}(t)$ for $B \in \mathcal{L}^{*}(\mathcal{H})$, we have

$$
\frac{d J}{d t}=\operatorname{Tr} B^{\dagger} \frac{d X^{2}}{d X} \frac{d X}{d t}=\operatorname{Tr} B^{\dagger}\left(2 X-C_{X}\right) \frac{d X}{d t} .
$$

Hence $d J / d X=\left(2 X-C_{X}\right)^{\dagger} B \in \mathcal{L}^{*}(\mathcal{H})$. In general, for $J=\operatorname{Tr} B^{\dagger} f(X)$, we have $d J / d X=(d f / d X)^{\dagger} B$.

For a multivariable functional $J\left(X_{1}, X_{2}, \ldots\right)$, the partial differential of $J$ with respect to $X_{i} \in \mathcal{L}(\mathcal{H})$ is defined in the same way by considering $\oplus_{i} \mathcal{L}(\mathcal{H})$, i.e., there exists a unique linear operator $A_{i} \in \mathcal{L}^{*}(\mathcal{H})$ such that

$$
\frac{d J}{d t}=\sum_{i} \operatorname{Tr} A_{i}^{\dagger} \frac{d X_{i}}{d t} .
$$

Then, the partial derivative is defined as $\partial J / \partial X_{i}=A_{i}$.

\section{B. $H$ functional}

Let us consider minimizing a real functional

$$
J(\mathbf{u})=S(X(T))
$$

subject to an operator equation

$$
\dot{X}(t)=f(X(t), \mathbf{u}(t)) \quad(0 \leqslant t \leqslant T),
$$

where $X=X^{\dagger} \in \mathcal{L}(\mathcal{H}), \mathbf{u} \in \mathbf{R}^{n}$ is a parameter to be determined to minimize $J$, and $f$ is an operator-valued function on $\mathcal{L}(\mathcal{H}) \times \mathbf{R}^{n}$.

Let $\mathbf{u}_{*}$ be a minimizing vector and suppose that

$$
\mathbf{u}_{\epsilon}(t)= \begin{cases}\mathbf{u}_{*}(t) & 0 \leqslant t<s-\epsilon, \quad s \leqslant t \leqslant T, \\ \mathbf{c} \neq \mathbf{u}_{*}(t) & s-\epsilon \leqslant t<s,\end{cases}
$$

and $X_{\epsilon}$ is the corresponding response. We first note that $X_{\epsilon}(s)=X(s)+\epsilon\left[f(X(s), \mathbf{c})-f\left(X(s), \mathbf{u}_{*}(s)\right)\right]+o(\epsilon)$. It follows from the chain rule that for $t>s$,

$$
\left.\frac{\partial X_{\epsilon}(t)}{\partial \epsilon}\right|_{\epsilon=0}=\left.G(t, s) \frac{\partial X_{\epsilon}(s)}{\partial \epsilon}\right|_{\epsilon=0},
$$

where $\{G(t, s) \mid s \leqslant t \leqslant T\}$ is a semigroup generated by $g=\partial f / \partial X \in \operatorname{Hy}(\mathcal{L})$ [37]. Hence $X_{\epsilon}(t)=X(t)+\epsilon Y(t)+o(\epsilon)$, where $\dot{Y}(t)=g(t) Y(t)$ with the initial condition $Y(s)=f(X(s), \mathbf{c})-f\left(X(s), \mathbf{u}_{*}(s)\right)$. 
Let us introduce the $H$ functional as

$$
H(X, P, \mathbf{u})=\operatorname{Tr} P f(X, \mathbf{u}),
$$

where $P=P^{\dagger} \in \mathcal{L}(\mathcal{H})$. Obviously $\quad \dot{X}=d H / d P$. Suppose that $P$ obeys $\dot{P}=-\partial H / \partial X$ with the transversality condition $P(T)=d S(X(T)) / d X$. Tr $P(t) Y(t)$ becomes a constant because the definition of the partial derivative reads $\dot{P}=-g^{\dagger}(t) P$. Thus, we have

$$
\begin{aligned}
\left.\frac{d J\left(\mathbf{u}_{\epsilon}\right)}{d \boldsymbol{\epsilon}}\right|_{\epsilon=0}= & \operatorname{Tr} P(T) Y(T)=\operatorname{Tr} P(s)[f(X(s), \mathbf{c}) \\
& \left.-f\left(X(s), \mathbf{u}_{*}(s)\right)\right] .
\end{aligned}
$$

Since $J\left(\mathbf{u}_{\epsilon}\right)$ is minimized at $\epsilon=0, d J /\left.d \epsilon\right|_{\epsilon=0} \geqslant 0$ for any $s$ and c. Thus, $\mathbf{u}_{*}$ minimizes $J$ if for any $\mathbf{u}(t) \in \mathbf{R}^{n}$,

$$
H\left(X, P, \mathbf{u}_{*}\right) \leqslant H(X, P, \mathbf{u}) .
$$

If the functional is of the form

$$
J(\mathbf{u})=S(X(T))+\int_{0}^{T} d t Q(X(t), \mathbf{u}(t))
$$

define an auxiliary variable $z(t)$ as $\dot{z}=Q(X, \mathbf{u})$ and set $\tilde{X}=X \oplus z, \tilde{f}=f \oplus Q$, and $\tilde{S}(\tilde{X})=S(X)+z$. Then, by considering the minimization of $J(\mathbf{u})=\tilde{S}(\tilde{X}(T))$ subject to a differential equation $\dot{\widetilde{X}}=\tilde{f}(\tilde{X}(t), \mathbf{u}(t))$, the same condition (16) can be obtained for the $H$ functional defined as $H=\operatorname{Tr} \widetilde{P} \widetilde{f}$, where $\widetilde{P}$ satisfies $\dot{\widetilde{P}}=-\partial H / \partial \tilde{X}$ with the transversality condition $\widetilde{P}(T)=d \tilde{S}(\tilde{X}) / d \tilde{X}$. This can be rewritten as $H=\operatorname{Tr} P f+r Q$, where $r \in \mathbf{R}$ satisfies $\dot{r}=-\partial H / \partial z=0$ and $r(T)=d \tilde{S} / d z=1$ so that $r=1$ for any $t \in[0, T]$. The $H$ functional is now given as

$$
H(X, P, \mathbf{u})=\operatorname{Tr} P f(X, \mathbf{u})+Q(X, \mathbf{u}) .
$$

One can see from operator derivatives that the effect of noncommutativity appears in this problem if the functional or the differential equation is nonlinear with respect to $X$.

\section{MODELS OF QUANTUM NETWORKS}

In this section, we introduce two models of quantum networks, spin-1/2 and bosonic systems, for which we formulate the optimal control problem of quantum walks.

\section{A. Spin-1/2 systems}

Let $G:=\left\{V_{G}, E_{G}\right\}$ be a graph with the set of vertices $V_{G}$ and its edges $E_{G}$. Two vertices $i, j$ are adjacent if $(i, j) \in E_{G}$. We associate a spin system with a graph $G$ by attaching a spin $1 / 2$ to each vertex. The system is then described by a Hilbert space $\mathcal{H}=\left[\mathbf{C}^{2}\right]^{\otimes \mid} V_{G} \mid$. The Hamiltonian of the system is defined by the form

$$
H(t)=H_{g}(t)+H_{l}(t) .
$$

$H_{g}$ represents global interactions between vertices and produces a quantum random walk on the graph. $H_{l}$ represents local interactions of control manipulations. For a structured manipulation, it can be written as $H_{l}(t)=H_{l}(\mathbf{u}(t))$, where $\mathbf{u}(t)=\left[u_{k}(t)\right]_{k \in V_{G}} \in \mathbf{R}^{\left|V_{G}\right|}$ is a control parameter.

The performance of quantum networks designed for a variety of purposes can be evaluated in a unified way by introducing cost functionals. Here, we consider two types of cost functionals. Let $X(t)$ be a density matrix of the spin system. The optimal control problem is defined to find $H_{l}$, which minimizes a functional

$$
\begin{gathered}
\text { (A) } J_{A}\left(H_{l}\right)=S(X(T), T) \quad\left(\left\|H_{l}(t)\right\| \leqslant m\right) \\
\text { (B) } J_{B}\left(H_{l}\right)=S(X(T), T)+\int_{0}^{T} d t Q\left(X(t), H_{l}(t)\right)
\end{gathered}
$$

subject to a differential equation

$$
\dot{X}(t)=f(X(t)) \quad(0 \leqslant t \leqslant T) .
$$

Here, $f$ is determined by the Hamiltonian (19). $S$ is a functional representing the terminal cost which reflects the purpose of the control. The cost functional (A) focuses on the effect of control only at time $T$. The constraint $\left\|H_{l}\right\| \leqslant m$ is necessary for the input to be finite. Sometimes this is not sufficient to produce a desirable performance. In the case of (B), a functional $Q$ is introduced as a running cost, which is usually chosen to improve the performance of the system, such as robustness. $Q$ is also involved in $H_{l}$ to balance the relative importance of the manipulation in the cost functional. It should be noted that the quantum effect appears if the functional is of the second or higher order with respect to $X$, as seen in Sec. II.

Let us consider an example in which a system is a $N$-spin chain with nearest neighbor interactions and the external field is applied to the center vertex to maximize entanglement between two specified vertices. The Hamiltonian is written as

$$
H^{s}=H_{g}^{s}+H_{l}^{s},
$$

$$
H_{g}^{s}=\sum_{i=-(N-1) / 2}^{(N-1) / 2} \frac{\beta}{4}\left(\sigma_{i}^{x} \sigma_{i+1}^{y}-\sigma_{i}^{y} \sigma_{i+1}^{x}\right),
$$

$$
H_{l}^{s}=\frac{u(t)}{2} \sigma_{0}^{z},
$$

where $\sigma_{i}^{x}, \sigma_{i}^{y}$, and $\sigma_{i}^{z}$ denote the Pauli matrices on the $i$ th vertex of the graph, $\beta$ is the interaction constant between the adjacent spins, and $u$ is a control parameter defined on the center vertex. We first note that the total $z$ component of the spin, $\sigma^{Z}=\Sigma_{i \in V_{G}} \sigma_{i}^{z}$, is invariant under $H^{s}$ since $\left[\sigma^{Z}, H^{s}\right]=0$. As a result, the Hilbert space $\mathcal{H}$ is decomposed into the direct sum of eigenspaces of $\sigma^{Z}$, i.e., $\mathcal{H}=\oplus_{l=0}^{N} \mathcal{H}_{l}$, where $\mathcal{H}_{l}$ is a Hilbert space of $l$ spins up. Suppose that the system is initially in $\mathcal{H}_{1}$. Then, the system is described by the natural basis 


$$
\Pi_{N}:=\left\{\left|\frac{N-1}{2}\right\rangle, \cdots,|0\rangle, \cdots,\left|-\frac{N-1}{2}\right\rangle\right\},
$$

where a unit vector $|k\rangle$ corresponds to the $k$ th vertex at which a spin is up.

Introducing Majorana operators

$$
a_{l}=\frac{1}{2} \prod_{i=-(N-1) / 2}^{l-1} \sigma_{i}^{z}\left(\sigma_{l}^{x}-i \sigma_{l}^{y}\right),
$$

we can rewrite the Hamiltonian (22) as

$$
H^{s}=\frac{i \beta}{2} \sum_{-(N-1) / 2}^{(N-1) / 2}\left(a_{l+1} a_{l}^{\dagger}-a_{l} a_{l+1}^{\dagger}\right)+u a_{0} a_{0}^{\dagger}=i \mathbf{a}\left[A^{s}+i u B^{s}\right] \mathbf{a}^{\dagger},
$$

where $\mathbf{a}:=\left[a_{(N-1) / 2} \cdots a_{-(N-1) / 2}\right]$ and

$$
A^{s}=\frac{\beta}{2}\left[\begin{array}{ccccc}
0 & 1 & 0 & \cdots & \\
-1 & 0 & 1 & & \\
0 & -1 & 0 & & \\
\vdots & & & \ddots & 1 \\
& & & -1 & 0
\end{array}\right], \quad B^{s}=\left[\begin{array}{lllll}
\ddots & & & & \\
& 0 & & & \\
& & 1 & & \\
& & 0 & \\
& & & \ddots
\end{array}\right] .
$$

Since the matrix expression of Hamiltonian $H^{s}$ with respect to $\Pi_{N}$ is equivalent to $A^{s}+i u B^{s}$ on the restricted subspace $\mathcal{H}_{1}$, Eq. (21) for this example is given by

$$
\dot{X}=\left(A^{s}+i u B^{s}\right) X+X\left(A^{s}+i u B^{s}\right)^{\dagger} .
$$

Note that this matrix expression is equivalent to a sequence of graphs consisting of two balanced binary trees of height $n$ with $2^{n}$ leaves [25].

To define the cost functional for entanglement generation, say between $n$th and $-n$th vertices, we consider steering the state as close as possible to a Bell state

$$
| \pm\rangle_{n}=\frac{1}{\sqrt{2}}(|n\rangle \pm|-n\rangle) .
$$

A feasible form of the terminal cost is $S(X)=\operatorname{Tr} \operatorname{Rg}(X)$, where $R=I-| \pm\rangle_{n}\langle \pm|$ and $g$ is an operator-valued function. If $g(X)=X, S$ is a complement to fidelity.

In case of the cost functional (B), $Q$ is conventionally chosen to suppress undesirable modes in the system and enable smooth control. In this example, the choice of $Q$ is particularly important because the system is oscillatory due to the unitary evolution. The simplest choice is given by the same functional as the terminal cost plus a constraint on the input, $Q(X, u)=\operatorname{Tr} \operatorname{Rg}(X)+m u^{2}(t)$. If $g(X)=X$, the optimal control maximizes the fidelity for the entire period while $u$ remains finite.

\section{B. Bosonic systems}

Quantum networks of bosonic systems are defined in the same way as the spin case. We associate a bosonic system with a graph $G=\left\{E_{G}, V_{G}\right\}$ by attaching an infinite dimensional Hilbert space to each vertex of the graph. Note that this example includes quantum networks of large spins in which a linearized Bloch sphere is regarded as a phase space. Thus, the bosonic system defined on the graph is also used as a quantum channel for quantum state transfer and entanglement generation between specified two vertices of the graph, provided that the performance of control is measured by the covariance of observables instead of the fidelity in this case.

Let $\mathbf{x}=\left[x_{1} \cdots x_{\mid V_{G}} y_{1} \cdots y_{\mid V_{G}}\right]^{T}$, where $x_{i}, y_{i}$ are the canonical observables on the $i$ th vertex and $X$ be the covariance matrix of the vector $\mathbf{x}$ with respect to a density matrix. The optimal control problem for the bosonic case is defined to minimize the functional, (A) or (B), subject to Eq. (21). Each term of the cost functional has the same role as the spin case so that our purpose is to find a control parameter that steers the state as close as possible to the target state while being finite. Note that $X$ is a covariance matrix here, whereas $X$ was a density matrix in the spin case.

We consider two types of bosonic examples: One is a case where each vertex is initially in an independent squeezed state and the system is subject to a Hamiltonian defined as

$$
\begin{gathered}
H_{1}^{b}=H_{g}^{b}+H_{l 1}^{b}, \\
H_{g}^{b}=\sum_{i=-(N-1) / 2}^{(N-1) / 2}\left[\frac{\alpha}{2}\left(x_{i}^{2}+y_{i}^{2}\right)+\frac{\beta}{2}\left(x_{i} y_{i+1}-y_{i} x_{i+1}\right)\right],
\end{gathered}
$$

$$
H_{l 1}^{b}=\frac{u(t)}{2}\left(x_{0}^{2}+y_{0}^{2}\right),
$$

where $\alpha, \beta$ are constants and $u$ is a control parameter at the center vertex. The other is initially in a vacuum state and subject to a Hamiltonian

$$
\begin{gathered}
H_{2}^{b}=H_{g}^{b}+H_{l 2}^{b}, \\
H_{l 2}^{b}=\frac{u(t)}{2}\left(x_{0} y_{0}+y_{0} x_{0}\right) .
\end{gathered}
$$

Equation (21) for $H_{i}^{b}(i=1,2)$ is expressed as

$$
\dot{X}=\left(A^{b}+u B_{i}^{b}\right) X+X\left(A^{b}+u B_{i}^{b}\right)^{\dagger}, \quad(i=1,2),
$$

where each matrix is given by

$$
\begin{gathered}
A^{b}=\left[\begin{array}{cc}
A^{s} & \alpha I \\
-\alpha I & A^{s}
\end{array}\right], \\
B_{1}^{b}=\left[\begin{array}{cc}
B^{s} \\
-B^{s}
\end{array}\right], \quad B_{2}^{b}=\left[\begin{array}{ll}
B^{s} & \\
& -B^{s}
\end{array}\right] .
\end{gathered}
$$

Note that the system is in a Gaussian state for all $t$ since the Hamiltonian is quadratic. If we consider entanglement generation between $n$th and $-n$th vertices, the performance is measured by Einstein-Podolsky-Rosen (EPR) uncertainty [38]

$$
\nu:=\frac{1}{2}\left[\Delta^{2}\left(x_{n}-x_{-n}\right)+\Delta^{2}\left(y_{n}+y_{-n}\right)\right],
$$

where $\Delta$ denotes a variance. If $\nu<1$, the pair contains EPR entanglement. The functional $S$ is then chosen as $S(X)$ 
$=\operatorname{Tr} \operatorname{Rg}(X)$, where, in the basis $\Pi_{N}, R=|-\rangle_{n}\langle-|\oplus|+\rangle_{n}\langle+| . Q$ is determined from physical requirements in the same way as the spin case.

\section{ENTANGLEMENT PROPAGATION}

Before considering the optimal control problems, we describe how entanglement is produced in the chain along the quantum random walk.

\section{A. Spin-1/2 systems}

We first consider the spin case where a spin at the center vertex is initially up and the rest is down and no control is applied. The spin state of the center vertex spreads over the chain along a quantum random walk represented by a Toeplitz matrix $A^{s}$ and produces entanglement between two vertices of the graph. Let us define a matrix $A^{c}$ as

$$
A^{c}=\frac{\beta}{2}\left[\begin{array}{ccccc}
0 & 1 & & & -1 \\
-1 & 0 & 1 & & \\
& -1 & 0 & & \\
& & & \ddots & 1 \\
1 & & & -1 & 0
\end{array}\right] .
$$

For $N \gg 1, A^{s}$ is equivalent to $A^{c}$ in the weak norm,

$$
\left|A^{s}-A^{c}\right|^{2}=\frac{\beta^{2}}{2 N} \rightarrow 0 \quad \text { as } N \rightarrow \infty,
$$

which allows us to approximate $A^{s}$ to $A^{c}$ for an infinite chain. The additional two elements in $A^{c}$ physically correspond to connecting both ends of the chain to each other as periodic boundary conditions.

$A^{c}$ is diagonalized by Fourier transform and the resulting eigenvalues and eigenvectors are given by

$$
\begin{gathered}
\lambda_{p}=-i \beta \sin p, \\
|p\rangle=\frac{1}{\sqrt{2 \pi}}\left[e^{i p N} e^{i p(N-1)} \cdots e^{-i p N}\right]^{T},
\end{gathered}
$$

where $p=2 \pi n / N(n=-(N-1) / 2, \ldots,(N-1) / 2)$. The matrix elements of a unitary operator $U(t)=e^{t A^{c}}$ are given by

$$
\begin{aligned}
U_{l m}(t)=\langle l|U| m\rangle & =\int_{-\pi}^{\pi} d p\langle l|U| p\rangle\langle p \mid m\rangle \\
& =\int_{-\pi}^{\pi} \frac{d p}{2 \pi} e^{i(l-m) p} e^{-i \beta t \sin p}=J_{l-m}(\beta t),
\end{aligned}
$$

where $J_{l-m}$ is a Bessel function of the first kind.

Let us examine entanglement between a symmetric pair, $n$th and $-n$th vertices, with a functional $\operatorname{Tr} R_{n}^{ \pm} X$, where $R_{n}^{ \pm}=| \pm\rangle_{n}\langle \pm|$, i.e., fidelity to Bell states. It follows from the matrix elements above that

$$
\operatorname{Tr} R_{n}^{ \pm} X(t)=\left[1 \pm(-1)^{n}\right] J_{n}^{2}(\beta t),
$$

where $J_{-n}=(-1)^{n} J_{n}$ has been used. It indicates that the type of entanglement between the pair depends on its parity. This tradeoff can be observed even in a system under control because the Lie group associated with the matrices $\left\{A^{s}, B^{s}\right\}$ remains the same property.

For a finite chain, the matrix element of the unitary operator can be calculated by directly diagonalizing the Toeplitz matrix $A^{s}$. Here, however, we use the multiple reflection to obtain the matrix element from the solution to the infinite case. Denote by $\widetilde{U}_{l m}$, the matrix element of the unitary operator for the finite case. Note that the unitary operator has to satisfy Eq. (21) with the following boundary and initial conditions:

$$
\begin{gathered}
\tilde{U}_{[(N+1) / 2] m}(t)=\tilde{U}_{-[(N+1) / 2] m}(t)=0, \\
\tilde{U}_{l m}(0)=\delta_{l m} .
\end{gathered}
$$

These conditions yield

$$
\tilde{U}_{l m}=\sum_{j=-\infty}^{\infty}\left[J_{l+2 j(N+1)-m}(\beta t)-J_{-l+(2 j-1)(N+1)-m}(\beta t)\right] .
$$

One can see that this matrix element has the same property as Bessel functions of the first kind

$$
\tilde{U}_{-n 0}=(-1)^{n} \widetilde{U}_{n 0} .
$$

As a result, the functional is given as

$$
\operatorname{Tr} R_{n}^{ \pm} X(t)=\left[1 \pm(-1)^{n}\right] \widetilde{U}_{n 0}^{2}(t),
$$

and the same properties as the infinite chain are concluded for the finite case.

\section{B. Bosonic systems}

In the bosonic example, we assume that the center vertex is initially squeezed instead of having no control applied and the rest is in a vacuum state. The Hamiltonian can be diagonalized in the same way as in the spin case. It is expressed in terms of creation and annihilation operators as

$$
H^{b}=\sum_{i}\left[\alpha a_{i}^{\dagger} a_{i}+\frac{i \beta}{2}\left(a_{i+1}^{\dagger} a_{i}-a_{i}^{\dagger} a_{i+1}\right)\right]=i \mathbf{a}\left(A^{c}+\alpha I\right) \mathbf{a}^{\dagger},
$$

where $\mathbf{a}:=\left[a_{(N-1) / 2} \cdots a_{-(N-1) / 2}\right]$. Hence, $\mathbf{a}(t)=U(t) \mathbf{a}(0)$, where $U=e^{t\left(A^{c}+\alpha I\right)}$ and its matrix element is given by

$$
U_{l m}(t)=J_{l-m}(\beta t) e^{i \alpha t} .
$$

To examine the nonlocal correlation between a symmetric pair, $n$th and $-n$th vertices, we assume that a covariance matrix of the center vertex is

$$
\left[\begin{array}{cc}
\Delta_{x} & 0 \\
0 & \Delta_{y}
\end{array}\right],
$$

where $\Delta_{x} \Delta_{y} \geqslant 1 / 4$. From the matrix element of the unitary operator, EPR complement between the two symmetric vertices defined as $\nu_{c}=1-\nu$ is given by 


$$
\nu_{c}=\left[1-\left(\Delta_{x}+\Delta_{y}\right)+(-1)^{n}\left(\Delta_{x}-\Delta_{y}\right) \cos 2 \alpha t\right] J_{n}^{2}(\beta t) .
$$

Obviously, there is the same tradeoff as the spin case. For simplicity, assume that $\alpha t \ll 1$. Then, the complement is given by

$$
\nu_{c}= \begin{cases}\left(1-2 \Delta_{x}\right) J_{n}^{2}(\beta t) & \text { if } n \text { is odd } \\ \left(1-2 \Delta_{y}\right) J_{n}^{2}(\beta t) & \text { if } n \text { is even } .\end{cases}
$$

If $\Delta_{x}\left(\Delta_{y}\right.$, respectively) $<1 / 2$, pairs of odd (even, respectively) sites are nonlocally correlated in proportion to the square of the Bessel functions, and both even and odd sites are not correlated at the same time. The same properties can be shown for the finite bosonic chain by replacing the Bessel function with the matrix element of $\widetilde{U}(t)$ as in the spin case.

These analyses provide significant information when we define a cost functional, because optimal control does not work at all for entanglement generation if we choose a wrong form of the cost functional.

\section{THE OPTIMAL CONTROL PROBLEM}

In this section, we consider the optimal control problems formulated in Sec. III and show numerical results.

\section{A. Spin-1/2 systems}

It is reasonable to choose the cost functional (A) if one wishes to maximize the effect of control at the terminal time. In this case, it can be shown that switching control is optimal. The $H$ functional is defined as

$$
H=\operatorname{Tr} P\left[\left(A^{s}+i u B^{s}\right) X+X\left(A^{s}+i u B^{s}\right)^{\dagger}\right] .
$$

The optimal input is determined from the condition (16) subject to the constraint $|u| \leqslant m$. Since the $H$ functional is linear with respect to $u$, it is minimized by

$$
u_{*}=-m \operatorname{sgn}\left[i \operatorname{Tr} P\left(B^{s} X-X B^{s}\right)\right],
$$

where

$$
\begin{aligned}
& \dot{X}=\frac{\partial H}{\partial P} \quad \text { [equivalent to Eq. (26)] } \\
& \dot{P}=-\frac{\partial H}{\partial X} \quad \text { with } P(T)=\frac{d S(X(T))}{d X} .
\end{aligned}
$$

As shown in Sec. IV, entanglement propagates as waves in the spin chain. This optimal control indicates that changing the input value $\pm m$ alternately at appropriate times effects a forced oscillation and the entanglement is maximized as the waves resonate in the chain. The optimal switching time for this amplification is provided from the above equations [39]. Figure 1(a) shows the numerical result of switching control for a cost functional $S=\operatorname{Tr} R X$.

Although the cost functional (A) works well to maximize the entanglement at the terminal time, there are difficulties in the experimental implementation of this control. In general, a state produced by switching control is fragile to uncertainties
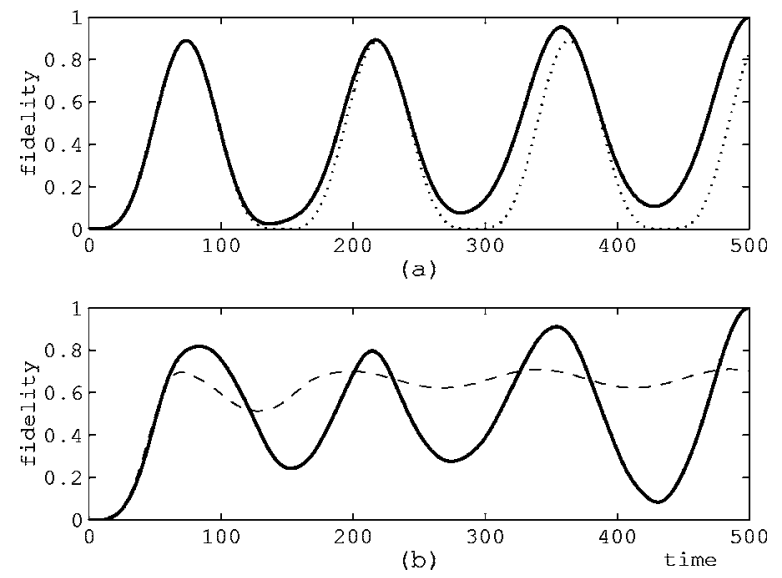

FIG. 1. Optimal control for producing entanglement between the two ends of the spin chain of five particles. (a) Fidelity to a Bell state subject to the functional (A) with a linear cost (solid line). The maximal fidelity is 0.99 at the terminal time. In the spin example, entanglement is produced without control (dotted line). (b) The same fidelity subject to the functional (B) with linear costs (dashed line) and quadratic costs (solid line). In the case of the quadratic costs, the maximal fidelity is 1.00 at the terminal time.

in the system. The control input is designed to amplify the oscillations so that the system is drastically sensitive to small changes in the parameters. A slightly different switching time or control value causes a cancellation of the waves and unexpected decoherence. For this reason, it is feasible to choose the cost functional (B) to control the system robustly.

A simple yet effective choice of the running cost is $Q=\operatorname{Tr} R X+m u^{2}$. The $H$ functional is then defined as

$$
H=\operatorname{Tr} P\left[\left(A^{s}+i u B^{s}\right) X+X\left(A^{s}+i u B^{s}\right)^{\dagger}\right]+Q .
$$

The condition (16) can be rewritten as

$$
\frac{\partial H}{\partial u}=0
$$

with the same constraint as the previous case

$$
\dot{X}=\frac{\partial H}{\partial P}, \quad \dot{P}=-\frac{\partial H}{\partial X} \quad \text { with } P(T)=\frac{d S(X(T))}{d X} .
$$

It is difficult to obtain analytical solutions to these equations in this case. Here, we apply the steepest descent method to find the extremal value $u_{*}$ of Eq. (51) subject to Eq. (52). Figure 1(b) shows the fidelity attained by the optimal control input under the cost functional (B). Although the performance of control at the terminal time is obviously lower than the switching control, the fidelity is constantly greater than $1 / 2$ for much of the time interval. Consequently, this control ensures robustness at the expense of the terminal performance. It would be difficult to enhance both performance and robustness because there is a tradeoff between them in general.

The optimal control considered above is classical in some sense because the dynamics of the system and the cost functional are linear with respect to $X$. Now we consider a cost functional of a higher order. The optimal input is determined 

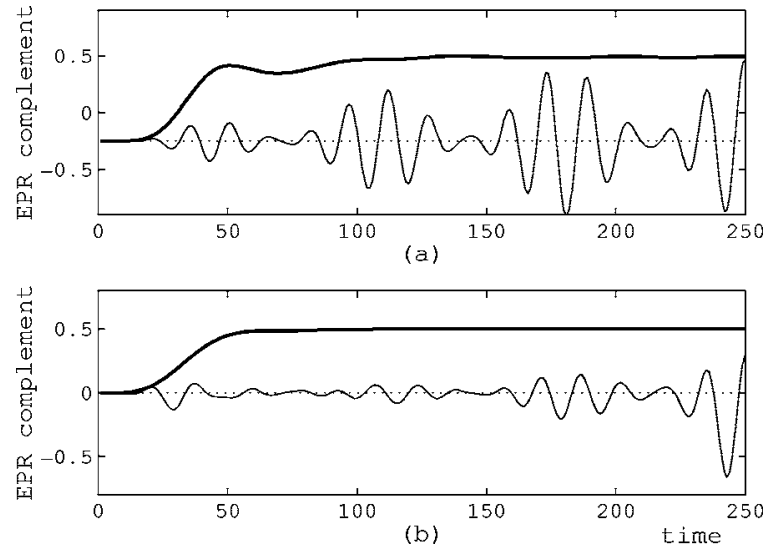

FIG. 2. Complement to EPR uncertainty between the two ends of the bosonic chain of five particles for $\alpha=0, \beta=1$ (bold line) and $\alpha=4, \beta=1$ (solid line). When the complement is greater than zero, the state involves EPR entanglement. (a) For Hamiltonian $H_{1}^{b}$, all particles are initially in the same minimum uncertainty squeezed state with $\Delta_{x}=0.25, \Delta_{y}=1$, and the system is controlled by a rotation of the center particle. (b) For Hamiltonian $H_{2}^{b}$, the system is in the vacuum state and controlled by squeezing the state of the center particle. In both cases, no correlation is produced without control (dotted line).

from their derivatives, as shown in Sec. II, and involves the noncommutativity of operators explicitly. This quantum effect of optimal control appears even in a simple cost functional. For instance, Fig. 1(b) shows the fidelity of the system optimized subject to the cost functional (B) with $S=\operatorname{Tr} R(X$ $\left.+X^{2}\right)$ and $Q=\operatorname{Tr} R\left(X+X^{2}\right)+m u^{2}$. A remarkable feature of this case is that the optimal input produces a perfect entanglement at the terminal time while maintaining relatively high fidelity during the process of control.

\section{B. Bosonic systems}

Let us consider a bosonic example described by Hamiltonian $H_{1}^{b}$ of Eq. (28). Suppose that all vertices are initially in identical and independent squeezed states. Unlike the spin examples above, no entanglement will be generated between any vertices without control because the initial state is a stationary solution. An optimal rotation control at the center vertex is designed to reconstitute the squeezing effect initially distributed in the system to maximize entanglement between two specified vertices. The procedure for this bosonic example is the same as in the spin case. Figure 2(a) shows the complement to the EPR uncertainty between the two ends of the chain subject to the cost functional (B) with $S=\operatorname{Tr} R\left(X+X^{2}\right)$ and $Q=\operatorname{Tr} R\left(X+X^{2}\right)+m u^{2}$. A distinctive feature of this case is that if the harmonic oscillation at each vertex is slow $\alpha \ll \beta$, the optimal control produces a steady entanglement (bold line). The wave nature of entanglement in the chain gradually appears with the increase of $\alpha$ and exhibits the beat pattern for $\alpha>\beta$, in which case, the entanglement is amplified along the beat.

In the case of Hamiltonian $H_{2}^{b}$ of Eq. (29), the squeezing effect locally produced at the center vertex spreads over the system along the quantum random walk and generates en- tanglement. Although the process is different from the previous case, we obtain similar numerical results, as shown in Fig. 2(b). We note that the maximal EPR complement is 0.5 in this example because of the tradeoff between two quadratures shown in Sec. IV. In the case of $\alpha \ll \beta$, the maximum is achieved by a constant input, and the optimality increases with the strength of squeezing at the center vertex.

\section{FURTHER APPLICATIONS}

In this section, we consider two applications of noncommutative optimal control. We first examine entanglement generation for the bosonic chain using another method of squeezing: measurement. The other application is quantum noise reduction in which a control input is designed to protect a quantum state from the influence of environments.

\section{A. Control via measurement}

We will consider entanglement generation for the bosonic chain by continuously detecting the state of the center vertex. Since the cost functional is given by the covariance matrix of the system, the optimization will be formulated as the design of a measurement process.

Assume that the system is initially in a Gaussian state with covariance matrix $X_{0}$ and the center vertex interacts with a reservoir described by a quantum Brownian motion [40], defined by an operator $a(t)$ satisfying

$$
\begin{gathered}
d a(t) d a^{\dagger}(t)=d t, \\
d a^{\dagger}(t) d a(t)=d a(t) d a(t)=0,
\end{gathered}
$$

where $d a(t)=a(t+d t)-a(t)$.

A system under measurement undergoes two processes: One is the evolution of the system described by a unitary operator

$$
U(t)=1+\int_{0}^{t}\left[l d a^{\dagger}-l^{\dagger} d a-\left(i H_{b}^{g}+\frac{1}{2} l^{\dagger} l\right) d t\right] U(t),
$$

where $H_{b}^{g}$ is given by Eq. (28b) and $l$ is a function of $x_{0}, y_{0}$. The other is a measurement process in which a single quadrature of the reservoir variable, say $a+a^{\dagger}$, is detected after the interaction with the system. These two processes are described, respectively, by

$$
\begin{gathered}
z(t)=U^{\dagger}(t) z U(t), \\
m(t)=U^{\dagger}(t)\left(a+a^{\dagger}\right) U(t)+k\left(b+b^{\dagger}\right),
\end{gathered}
$$

where $z$ is an arbitrary system operator and $b$ is an additional quantum and/or classical Brownian motion uncorrelated with $a$, and $k$ is a constant. If the system operator $l$ is linear in $x_{0}, y_{0}$, then Eq. (55) can be rewritten as linear equations

$$
\begin{gathered}
d \boldsymbol{x}=A \boldsymbol{x} d t+B d \xi, \\
d \boldsymbol{m}=C \boldsymbol{x} d t+D d \xi,
\end{gathered}
$$

where $\mathbf{x}=\left[x_{i} y_{i}\right]_{i \in V_{G}}^{T}$ is a vector of two quadratures at all vertices; $\boldsymbol{\xi}$ is a vector of $a, b$ defined in the same way as $\mathbf{x}$; and 
$A, B, C$, and $D$ are constant matrices. Because of linearity, the system remains the Gaussian property so that there is no difference between classical and quantum systems in their stochastic properties [41]. As a result, all quantum variables $\mathbf{x}, \boldsymbol{\xi}$ can be interpreted classically and the measurement process is described by the classical conditional expectation.

The conditional expectation of $\mathbf{x}$ given the process Eq. (56) is defined by a unique random variable $\hat{\mathbf{x}}$ that is a functional of $\mathbf{m}$ and satisfies the orthogonality condition $\mathrm{E}[(\mathbf{x}$ $-\hat{\mathbf{x}}) \mathbf{f}(\mathbf{m})]=0$ for any function $\mathbf{f}$. By definition, $\hat{\mathbf{x}}$ turns out to be a random variable which minimizes the cost functional $\operatorname{Tr} R X$, where $X=\mathrm{E}[\mathbf{x}-\mathbf{f}(\mathbf{m})]^{2}$, i.e., $\hat{\mathbf{x}}=\arg \min _{f} \operatorname{Tr} R X$ and the resulting $X$ becomes the covariance matrix of the system under measurement.

To find the covariance matrix of the system under measurement, suppose an innovation process $d \mathbf{f}=A \mathbf{f} d t$ $+L(d \mathbf{m}-C \mathbf{f} d t)$, where $L$ is determined to minimize the cost functional. It follows from Eq. (53) that $\dot{X}=(A-L C) X$ $+(A-L C)^{\dagger}+(B-L D)(B-L D)^{\dagger}:=h$. In this case, the $H$ functional is defined as $H=\operatorname{Tr}[R X+P h]$ for some $P$. Then, the optimal cost is given by the same condition as Eq. (16), i.e., $\partial H / \partial L=0$. It follows from operator derivatives that $L=\left(X C^{\dagger}+B D^{\dagger}\right)\left(D D^{\dagger}\right)^{-1}$. Hence the covariance matrix is given by

$$
\dot{X}=A X+X A^{\dagger}+B B^{\dagger}-V\left(D D^{\dagger}\right)^{-1} V^{\dagger},
$$

where $V=X C^{\dagger}+B D^{\dagger}$ and $X(0)=X_{0}$.

From Eq. (57), it turns out that entanglement generation is optimized by the operator $l$ which represents the interaction between the system and the reservoir and, hence, determines the type of measurement. The optimal control problem is then defined by the minimization of the cost functional (A) or (B) with respect to $l$.

In the case of entanglement generation via measurement, the optimality is sensitive to the measurement efficiency determined by the additional noise $b$. In general, $l$ becomes a function of $X$ after optimization. Here, however, to see the relationship between the optimal interaction and the measurement efficiency, we consider a simplified case in which $l$ is of the form $l=x_{0}+i c y_{0}$, where $c$ is a real constant to be adjusted to maximize the EPR entanglement. Note that $c$ $=0$ corresponds to quantum nondemolition (QND) measurement of $x_{0}$. From the analysis of Sec. V, QND measurement is expected to be optimal for entanglement generation because it has the strongest effect of squeezing. This is actually true if $k=0$, i.e., no additional noise disturbs the measurement outcome and the measurement efficiency is unity.

Figure 3 shows the relation between $k$ and the optimal $c$ for entanglement generation between the two ends of the chain at a certain terminal time. When $k=0$, QND measurement produces the maximal entanglement. However, QND measurement is not necessarily optimal if the measurement process is disturbed by the additional noise.

\section{B. Quantum noise reduction}

The process of quantum optimal control considered so far consists of three elements: plant, controller, and communica-

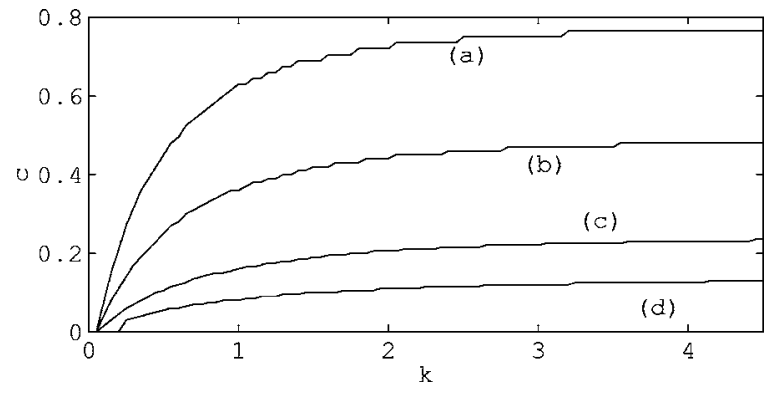

FIG. 3. Entanglement generation via measurement for the bosonic chain of five particles. Optimal measurement parameter $b$ for the terminal time (a) $T=2$, (b) $T=4$, (c) $T=10$, (d) $T=100$.

tion line. The plant is the state of the target vertices, which was both ends of the chain in the examples of entanglement generation. The controller is the manipulation described by the local Hamiltonian, designed to steer the plant to a desired state. The communication line is the quantum random walk between the plant and the controller and is expressed by an uncontrollable global Hamiltonian.

This system can be analyzed in a different way from the control theoretical perspective. Suppose that the plant initially contains entanglement and interacts with the quantum random walk on a graph of a large number of vertices. Although the whole system is conservative and time reversible, coupling with the random walk causes the dynamics of the plant to appear to be stochastic and irreversible over a finite time horizon. The quantum random walk can be thought of as a noise disturbing the plant, and the entanglement in the plant is lost due to large degrees of freedom. Then, the controller is utilized to protect the entanglement from the noise over the finite time horizon. The basic idea is illustrated in Fig. 4. Our purpose is to design the controller to effectively isolate the plant from the noise and reduce the influence of dissipation on the plant. This is called a noise reduction problem.

The effect of the quantum random walk on the plant is examined by eliminating irrelevant variables via a standard procedure such as the projection operator formalism for deriving the generalized Langevin equation $[42,43]$ and model reduction based on the input-output relation of the plant [44]. In fact, it can be shown that the Langevin equation of the spin system is equivalent to the equation of motion for the

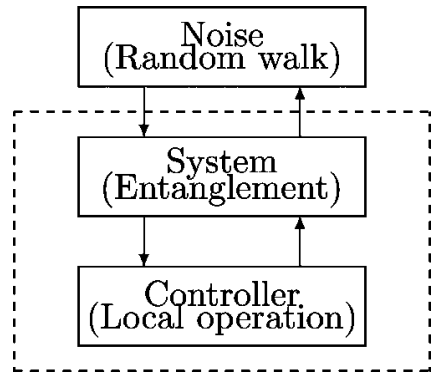

FIG. 4. Schematic representation of quantum noise reduction. The role of the controller is to isolate the dotted frame from environmental systems by reducing undesirable influences of the noise as far as possible. 


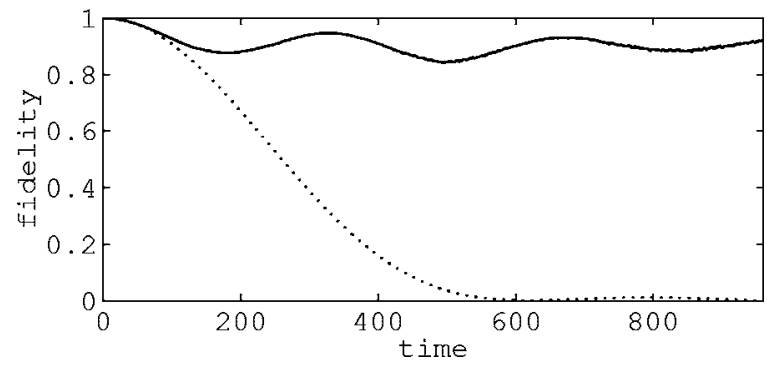

FIG. 5. Quantum noise reduction for the spin chain of nine particles. The two ends of the chain are initially in a Bell state and independent of the rest. If no control is applied, the noise causes the dissipation of entanglement over the finite time horizon (dotted line). The initially shared entanglement is protected by a rotation of each particle (solid line).

velocity autocorrelation function of a classical harmonic chain. Although we do not consider these methods here, it is worth noting that model reduction is useful in evaluating the performance of noise reduction.

We assume that the initial state of the plant is known and to be kept as long as possible. The noise reduction problem is then to find a controller which protects the initial state from the noise. The performance of the controller, in this case, may be measured by a distance between quantum states over a finite time interval. Here, we define a cost functional as

$$
J(u)=\int_{0}^{T} d t\left[\|X(0)-X(t)\|^{2}+m u^{2}\right]
$$

where $\|\cdot\|$ is a norm, e.g., the Hilbert-Schmidt norm. The optimal control input is obtained through the same procedure with the $H$ functional

$$
H=\operatorname{Tr} P f(X)+\|X(0)-X(t)\|^{2}+m u^{2} .
$$

Note that if the initial state is pure, this cost functional is effectively equivalent to fidelity. However, the optimization process is involved in the noncommutativity of the states at different times because of the quadratic term.

Figure 5 is a numerical result for the spin-1/2 chain in which the plant, both ends of the chain, is initially prepared in a Bell state. If no control is applied, the entanglement initially stored in the plant slowly dissipates into large degrees of freedom, as depicted in the dotted line. In contrast, the solid line shows that the controller effectively protects the plant from the noise and a high fidelity is maintained over the finite time interval.

\section{CONCLUSION}

We have developed optimal control theory based on operator derivatives which is explicitly involved in the noncommutativity of operators. This method of control has been applied to entanglement generation for both spin and bosonic networks and quantum noise reduction. Although we confined investigation to specific types of quantum networks in this paper, the formulation of noncommutative optimal control has a wide range of applications.
[1] A. S. Holevo, Probabilistic and Statistical Aspects of Quantum Theory (North-Holland, Amsterdam, 1982).

[2] C. W. Helstrom, Quantum Detection and Estimation Theory (Academic Press, New York, 1976).

[3] M. A. Nielsen and I. L. Chuang, Quantum Computation and Quantum Information (Cambridge University Press, Cambridge, 2000).

[4] G. M. Huang, T. J. Tarn, and J. W. Clark, J. Math. Phys. 24, 2608 (1983).

[5] A. P. Peirce, M. A. Dahleh, and H. Rabitz, Phys. Rev. A 37, 4950 (1988).

[6] I. H. Deutsch and P. S. Jessen, Phys. Rev. A 57, 1972 (1998).

[7] S. Lloyd, Phys. Rev. A 62, 022108 (2000).

[8] D. D'Alessandro, J. Math. Phys. 42, 4488 (2001).

[9] N. Khaneja, S. J. Glaser, and R. Brockett, Phys. Rev. A 65, 032301 (2002).

[10] L. K. Thomsen, S. Mancini, H. M. Wiseman, J. Phys. B 35, 4937 (2002).

[11] C. Rangan, A. M. Bloch, C. Monroe, and P. H. Bucksbaum, Phys. Rev. Lett. 92, 113004 (2004).

[12] D. A. Steck, K. Jacobs, H. Mabuchi, T. Bhattacharya, and S.
Habib, Phys. Rev. Lett. 92, 223004 (2004).

[13] R. van Handel, J. K. Stockton, and H. Mabuchi, IEEE Trans. Autom. Control 50, 768 (2005).

[14] T. Assion, M. Baumert, T. Brixner, B. Kiefer, V. Seyfrien, M. Strehle, and G. Gerber, Science 282, 919 (1998).

[15] R. J. Levis, G. M. Menkir, and H. Rabitz, Science 292, 709 (2001).

[16] J. L. Herek, W. Wohleben, R. J. Cogdell, D. Zeidler, and M. Motzkus, Nature (London) 417, 533 (2002).

[17] N. V. Morrow, S. K. Dutta, and G. Raithel, Phys. Rev. Lett. 88, 093003 (2002).

[18] B. Julsgaard, J. Sherson, J. I. Cirac, J. Fiurasek, and E. S. Polzik, Nature (London) 432, 482 (2004).

[19] J. McKeever, A. Boca, A. D. Boozer, R. Miller, J. R. Buck, A. Kuzmich, and H. J. Kimble, Science 303, 1992 (2004).

[20] J. M. Geremia, J. K. Stockton, and H. Mabuchi, Science 304, 270 (2004).

[21] M. Yanagisawa and H. Kimura, IEEE Trans. Autom. Control 48, 2107 (2003).

[22] M. R. James, Phys. Rev. A 69, 032108 (2004).

[23] W. Fleming and R. Rishel, Deterministic and Stochastic Opti- 
mal Control (Springer-Verlag, New York, 1975).

[24] E. Farhi and S. Gutmann, Phys. Rev. A 58, 915 (1998).

[25] A. Childs, E. Farhi, and S. Gutmann, Quantum Inf. Process. 1, 35 (2002).

[26] A. M. Childs, R. Cleve, E. Deotto, E. Farhi, S. Gutmann, and D. A. Spielman, e-print quant-ph/0209131 (unpublished).

[27] L. Amico, A. Osterloh, F. Plastina, R. Fazio, and G. M. Palma, Phys. Rev. A 69, 022304 (2004).

[28] V. Subrahmanyam, Phys. Rev. A 69, 034304 (2004)

[29] T. J. Osborne and N. Linden, Phys. Rev. A 69, 052315 (2004).

[30] J. Eisert, M. B. Plenio, S. Bose, and J. Hartley, Phys. Rev. Lett. 93, 190402 (2004).

[31] C. Albanese, M. Christandl, N. Datta, and A. Ekert, Phys. Rev. Lett. 93, 230502 (2004).

[32] M. B. Plenio and F. L. Semião, New J. Phys. 7, 73 (2005).

[33] S. Bose, Phys. Rev. Lett. 91, 207901 (2003).

[34] M. Christandl, N. Datta, A. Ekert, and A. J. Landahl, Phys. Rev. Lett. 92, 187902 (2004).
[35] E. Hille and R. S. Phillips, Functional Analysis and SemiGroup (American Mathematical Society, Providence, RI, 1948).

[36] K. Deimling, Nonlinear Functional Analysis (Springer-Verlag, New York, 1985).

[37] E. A. Coddington and N. Levinson, Theory of Ordinary Differential Equations (McGraw-Hill, New York, 1955).

[38] G. Giedke, M. M. Wolf, O. Krüger, R. F. Werner, and J. I. Cirac, Phys. Rev. Lett. 91, 107901 (2003).

[39] R. R. Mohler, Bilinear Control Processes (Academic Press, New York, 1973).

[40] K. R. Parthasarathy, An Introduction to Quantum Stochastic Calculus (Birkhäuser, Boston, 1992).

[41] E. Wigner, Phys. Rev. 40, 749 (1932).

[42] R. Zwanzig, J. Chem. Phys. 33, 1338 (1960).

[43] H. Mori, Prog. Theor. Phys. 33, 423 (1965).

[44] M. Yanagisawa, ISCIE Journal (to be published). 